\title{
Soot reference materials for instrument calibration and intercomparisons: a workshop summary with recommendations
}

\author{
D. Baumgardner ${ }^{1}$, O. Popovicheva ${ }^{2}$, J. Allan ${ }^{3}$, V. Bernardoni ${ }^{4}$, J. Cao ${ }^{5}$, F. Cavalli ${ }^{6}$, J. Cozic ${ }^{7}$, E. Diapouli ${ }^{8}$, \\ K. Eleftheriadis ${ }^{8}$, P. J. Genberg ${ }^{9}$, C. Gonzalez ${ }^{10}$, M. Gysel ${ }^{11}$, A. John ${ }^{12}$, T. W. Kirchstetter ${ }^{13}$, T. A. J. Kuhlbusch ${ }^{12,14}$, \\ M. Laborde ${ }^{11}$, D. Lack ${ }^{15,16}$, T. Müller ${ }^{17}$, R. Niessner ${ }^{18}$, A. Petzold ${ }^{19}$, A. Piazzalunga ${ }^{20}$, J. P. Putaud ${ }^{6}$, J. Schwarz ${ }^{15,16}$, \\ P. Sheridan ${ }^{15}$, R. Subramanian ${ }^{21}$, E. Swietlicki ${ }^{9}$, G. Valli ${ }^{4}$, R. Vecchi ${ }^{4}$, and M. Viana ${ }^{22}$ \\ ${ }^{1}$ Centro Ciencias de la Atmósfera, Universidad Nacional Autónoma de México, Mexico City, Mexico \\ ${ }^{2}$ Nuclear Physics, Moscow, State University, Moscow, Russia \\ ${ }^{3}$ University of Manchester, Manchester, UK \\ ${ }^{4}$ Department of Physics, Università degli Studi di Milano \& National Institute of Nuclear Physics, Milan, Italy \\ ${ }^{5}$ Institute of Earth Environment, Chinese Academy of Sciences, Xian, China \\ ${ }^{6}$ European Commision - Joint Research Centre - Institute for Environment and Sustainability, Ispra, Italy \\ ${ }^{7}$ Laboratory of Glaciology and Geophysics of the Environment, St Martin d'Hères Cedex, France \\ ${ }^{8}$ E.R.L, Institute of Nuclear Technology \& Radiation Protection, N.C.S.R. Demokritos, 15310, Attiki, Greece \\ ${ }^{9}$ Div. of Nuclear Physics, Lund University, P.O. Box 118, 22100, Lund, Sweden \\ ${ }^{10}$ National Institute of Standards and Technology, Gaithersburg, MD, USA \\ ${ }^{11}$ Laboratory of Atmospheric Chemistry, Paul Scherrer Institut, Villigen, Switzerland \\ ${ }^{12}$ IUTA e.v., Air Quality \& Sustainable Nanotechnology Unit, Duisburg, Germany \\ ${ }^{13}$ Lawrence Berkeley National Laboratory, Berkeley, CA, USA \\ ${ }^{14}$ King's College London, Environmental Research Group, London, UK \\ ${ }^{15}$ Earth Systems Research Laboratory, National Oceanic and Atmospheric Administration, Boulder, CO, USA \\ ${ }^{16}$ Cooperative Institute for Research in Environmental Sciences, University of Colorado, Boulder, CO, USA \\ ${ }^{17}$ Leibniz Institute for Tropospheric Research, Leipzig, Germany \\ ${ }^{18}$ Technische Universität, München, Germany \\ ${ }^{19}$ Deutschen Zentrums für Luft- und Raumfahrt, Oberpfaffenhofen, Germany \\ ${ }^{20}$ Department of Environmental and Territorial Sciences, University of Milan-Bicocca \& Department of Inorganic, \\ Metallorganic and Analytical Chemistry, Università degli Studi di Milano, Milan, Italy \\ ${ }^{21}$ RTI, Research Triangle, NC, USA \\ ${ }^{22}$ IDAEA-CSIC: Institute for Environmental Assessment and Water Research (IDÆA), Spanish Research Council - CSIC, \\ Barcelona, Spain
}

Correspondence to: D. Baumgardner (darrel.baumgardner@gmail.com)

Received: 24 February 2012 - Published in Atmos. Meas. Tech. Discuss.: 19 March 2012

Revised: 29 June 2012 - Accepted: 29 June 2012 - Published: 2 August 2012

\begin{abstract}
Soot, which is produced from biomass burning and the incomplete combustion of fossil and biomass fuels, has been linked to regional and global climate change and to negative health problems. Scientists measure the properties of soot using a variety of methods in order to quantify source emissions and understand its atmospheric chemistry, reactivity under emission conditions, interaction with solar radia-
\end{abstract}

tion, influence on clouds, and health impacts. A major obstacle currently limiting progress is the absence of established standards or reference materials for calibrating the many instruments used to measure the various properties of soot.

The current state of availability and practicability of soot standard reference materials (SRMs) was reviewed by a group of 50 international experts during a workshop in June 
of 2011. The workshop was convened to summarize the current knowledge on soot measurement techniques, identify the measurement uncertainties and limitations related to the lack of soot SRMs, and identify attributes of SRMs that, if developed, would reduce measurement uncertainties. The workshop established that suitable SRMs are available for calibrating some, but not all, measurement methods. The community of users of the single-particle soot-photometer (SP2), an instrument using laser-induced incandescence, identified a suitable SRM, fullerene soot, but users of instruments that measure light absorption by soot collected on filters did not. Similarly, those who use thermal optical analysis (TOA) to analyze the organic and elemental carbon components of soot were not satisfied with current SRMs. The workshop, and subsequent, interactive discussions, produced a number of recommendations for the development of new SRMs, and their implementation, that would be suitable for the different soot measurement methods.

\section{Introduction}

Soot, a product of incomplete combustion of any carbon containing fuels under hot and air-starved conditions, is a constituent of atmospheric aerosol particles that has received the attention of the climate and health research communities because of the impact of soot-containing aerosol on modifying radiative fluxes and increasing the susceptibility to diseases that affect the respiratory, cardiovascular and nervous systems of humans. The term soot is used by the Intergovernmental Panel on Climate Change to denote any light-absorbing, combustion-generated aerosols (IPCC, 1996) whereas black carbon (BC) refers to the optical properties of soot (e.g. Bond and Bergstrom, 2006, and references therein). We will use soot from here onward to indicate that we are interested in all of the properties of this type of aerosol particle, not just those related to light absorption. The general term "soot" refers to combustion-generated aerosol mixtures of two components: first, the most thermally refractory and light absorbing byproduct of incomplete combustion, which is commonly referred to as elemental carbon (EC; thermal/optical analysis) or black carbon (BC; light absorption methods); and second, organic carbon (OC) that can have a wide range of thermal and light-absorbing properties. Here we use "soot" to indicate the whole range of particles produced under the conditions described above, not merely EC or OC within such particles.

Although most countries that routinely measure air quality have established standards related to the mass of particulate matter below a diameter of $10 \mu \mathrm{m}\left(\mathrm{PM}_{10}\right)$ or $2.5 \mu \mathrm{m}\left(\mathrm{PM}_{2.5}\right)$, very few have regulations related to acceptable levels of EC. It is interesting to note that the European Union (EU) has had a requirement to monitor elemental carbon (EC) in $\mathrm{PM}_{2.5}$ at selected rural background sites since mid-2010 (Eu- ropean Council Directive 2008/50/EC). There is a technical report (CEN/TR 16243, 2011) on thermal optical transmittance/reflectance methods for measuring EC and OC, wherein thermal protocols (i.e. NIOSH-like, NIOSH5040, IMPROVE, and EUSAAR_2) are recommended.

The properties of atmospheric soot, i.e. its sizes, shapes, concentrations, absorption and scattering coefficients and chemical composition, are complex and can vary greatly, depending on many environmental factors. Many instruments have been developed for measuring some of these properties, e.g. the light scattering and absorption at different wavelengths and the concentration of organic and elemental carbon (OC and EC). Significant progress has been made in the development of these sensors but obstacles common to almost every technique remain. One obstacle is the lack of soot standard reference materials (SRMs) with properties specific to those that the instruments are designed to measure, and, optimally, are representative of soot found in the natural environment. The lack of SRMs limits comparisons between different instruments and methods.

In 1999, a steering committee was formed to make recommendations for representative and acceptable BC reference materials for the environmental sciences community. This committee was made up of experts from the soil sciences community and hence their focus was primarily on the characterization of $\mathrm{BC}$ in soil, rivers, lakes and marine environments. Their recommendations are reported by Schmidt et al. (2003) where they also underscore the gravity of methodological problems, using as an example a study on a set of soils which showed a factor of 500 difference between $\mathrm{BC}$ concentrations measured with a range of techniques (Schmidt et al., 2001). The recommendations included (i) five matrices containing BC (soot, charcoal, aerosol, soil, and sediment), and (ii) five materials potentially creating BC during analysis for use in detecting methodological artifacts (the complete list of recommendations can be found at http://www.geo.unizh.ch/phys/bc). None of the materials that were recommended, however, meet the requirements that have been established by international standardization agencies (see below). The actions taken by the Steering Committee for $\mathrm{BC}$ reference materials, while a positive step, have not produced a noticeable movement in the atmospheric sciences community, i.e. those doing air quality, climate and health research, towards establishing a consensus with respect to soot SRMs.

An explanation for the lack of progress in developing SRMs in the environmental sciences community is beyond the scope of this review article; however, a significant number of those in this community who do soot research have recognized for some time the need to take definitive action to rectify this situation. Hence, in June 2011, a workshop was convened, following the 10th International Conference of Carbonaceous Particles in the Atmosphere (ICCPA) in Vienna, Austria, to address the issues related to finding SRMs suitable for calibrating, validating and comparing 
instruments that measure soot properties. The one-day workshop focused on: (1) reviewing the current state of technology for measuring some of the properties of soot, (2) identifying potential candidates for SRMs and (3) formulating a viable plan for moving the state of soot measurement science forward through the development of SRMs that will be used by the environmental sciences community.

The workshop consisted of two sessions: (1) keynote presentations that reviewed the technology and defined the issues related to the measurement of soot properties, and (2) working group discussions of solutions to the measurements problems and recommendations to implement them. The remainder of this paper summarizes the keynote presentations and the findings and recommendations of the 50 participants that attended the meeting. The discussions from the workshop have been supplemented by the co-authors of this paper in order to clarify and provide sufficient detail to understand the issues. The following section summarizes the current state of the instrumentation for measuring soot properties, the techniques for calibrating and validating the technology and the major challenges that must be addressed. The third section reviews the currently available materials that could serve as SRMs for the different measurement techniques, and the final section is the summary and recommendations.

Note: a number of issues related to measurement techniques, interpretation of the results, calibration methodology, etc. are under continuing discussion. So that there is no presumption that all of the material presented here meets with the complete acceptance of those who are co-authoring this article, sections that are written in quotations indicate material that might not reflect $100 \%$ agreement of the participants.

\section{Calibration and validation of soot measurement techniques}

Soot has many physical (size, shape, concentration, thermal behavior, mixing state, and solubility), optical (light scattering and absorption) and chemical (OC, EC, inorganics, metals and mineral impurities) properties that should be measured for complete characterization. During the workshop, the focus was on filter-based and photoacoustic techniques for measuring the light absorption coefficient, on a laserinduced incandescence technique that measures the mass concentrations of refractory black carbon (rBC) and on thermal optical analysis techniques to derive OC and EC. In the following sections these measurement techniques are briefly described, including their uncertainties and limitations.

\subsection{Challenges to light absorption measurements}

The most common methods for determining the light absorbing properties of soot use measurements of light transmitted through an aerosol-loaded filter or the sound waves created as soot particles increase the temperature in a sample chamber as they absorb light from an oscillating-intensity laser (photoacoustic techniques). Emerging technologies, such as cavity ring-down (e.g. Thompson et al., 2008), were not discussed at the workshop because no representative from that community of users attended the meeting.

The issue of converting light absorption to a mass concentration of $\mathrm{BC}$ was not discussed because of the many problems that are associated with arriving at an appropriate specific absorption cross-section with which to do this conversion. This problem has been discussed at length in many publications, i.e. Fuller (1994, 1995), Fuller et al. (1999) and Bond and Bergstrom (2006).

\subsubsection{Light transmission methods}

In general, instruments that use light transmission are: (1) relatively inexpensive to purchase and maintain; (2) small, lightweight and portable; (3) easy to operate; and (4) suitable for long-term unattended operation (some models). In theory, the design is based on the assumption that the instrument's response to light absorbing aerosols is well understood and relatable to "reference" absorption methods. In practice, particles are continuously deposited on a white filter, while the reduction in light transmittance (caused by the growing deposit of particles) through the filter is continuously monitored and related to the transmittance through a clean portion of the filter. The component of the attenuation caused by the light absorption by particles on the filter is determined by applying empirical calibration schemes or radiative transfer models of the combined filter and aerosol system. All of the filter-based methods rely on some sort of radiative transfer model to invert their data, although this is hidden from the user in consumer-grade instruments. The commercial instruments provide a value for the absorption coefficient, attenuation or black carbon mass concentration. It is generally accepted that additional correction factors are needed to take into account particle loading, light scattering, etc. There are numerous publications on the measurement and analysis techniques, e.g. Bond et al. (1999), Weingartner et al. (2003), Virkkula et al. (2005, 2007), Virkkula (2010), Arnott et al. (2005), Petzold et al. (2005) and Ogren (2010).

The major issues and questions, not necessarily independent, associated with the light transmission measurement technique that potentially could be addressed with SRMs are as follows:

- The absorption coefficient is the desired property but attenuation is what is actually measured; hence, does light absorption of aerosols on a filter truly represent aerosol light absorption in the atmosphere (i.e. suspended state)?

- What is the magnitude of the artefact produced by multiple scattering of the particles in the filter matrix as a 
function of particle size, shape, refractive index, single scattering albedo (SSA), concentration, and history of the filter loading?

- Given that most instruments that measure light transmission require multiple correction schemes, which correction is best suited for a range of different types of aerosols?

- How can results be scaled to different wavelengths?

- How will the choice of SRM influence the results?

- Does a particle's size-dependent sensitivity (e.g. Lack et al., 2009; Nakayama et al., 2010; Moteki et al., 2010) influence the calibration?

- How do we account for changes in light absorption with changing relative humidity? Is this an issue that could be addressed with an SRM?

- What is the potential bias due to inorganic and organic aerosol (Lack et al., 2008) and liquid droplets contributions to the attenuation (e.g. yellow beads observed by Subramanian et al., 2007)?

- What is the magnitude of correction needed for the effects of filter flexing/settling (from internal pressure/flow variations)?

The various correction schemes that are currently being applied can partially account for some problems and issues listed above; however, the magnitude of each effect is difficult to determine since many of the effects occur at the same time and superimpose themselves on one another. It is anticipated that with the proper choice of SRMs, the magnitude of the various artefacts can be evaluated as well as the efficiency of the schemes to correct for them.

As previously noted, numerous methods have been developed to correct for biases introduced by the artifacts and to decrease the measurement uncertainties. The goal of a correction method is to find a unique relation between the instrumental measurement signal(s) and the absorption coefficient of airborne particles. This approach requires an understanding of the variables that influence the signals, not only the optical, mechanical and electrical limitations of the sensor, but also parameters that are related to the particle properties, e.g. ambient conditions and other atmospheric components such as relative humidity and condensing/evaporating gases. A detailed analysis is required to estimate the sensitivity of the measurement system to these parameters. Corrections for particle loading and light scattering were introduced by Bond et al. (1999) followed by additional refinements proposed by Weingartner et al. (2003), Virkkula et al. $(2005,2010)$ and Ogren (2010). A more sophisticated correction was submitted by Petzold and Schönlinner (2004), who used a four-stream radiative transfer model to take into account the contributions to the attenuation and reflectance

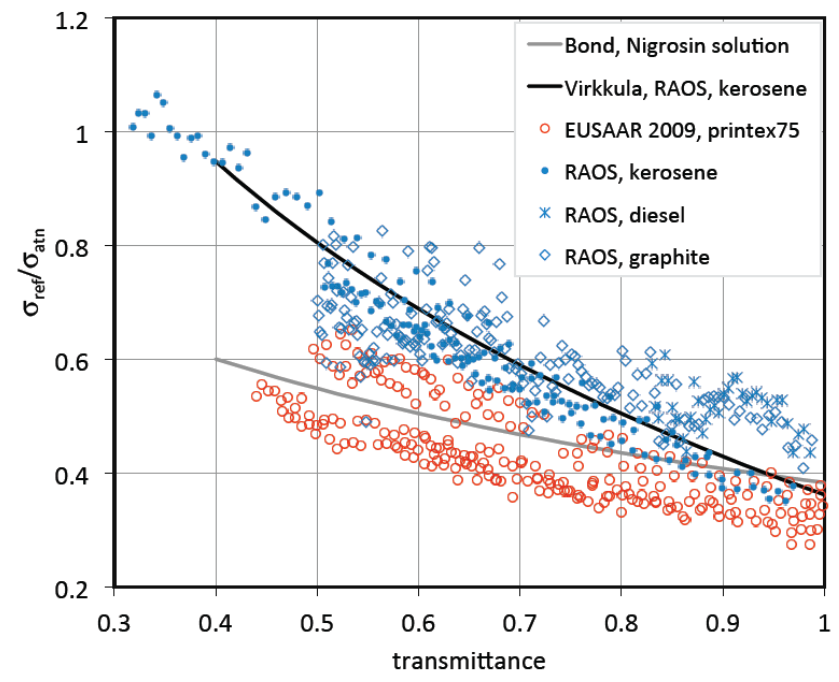

Fig. 1. Data measured during two absorption photometer workshops (EUSAAR 2009, and RAOS) for different types of Aerosols. Correction schemes developed by Bond et al. (1999) and Virkkula et al. $(2005,2010)$ are shown as solid lines.

by the light scattering from the particles on the filter. This requires multiple iterations that yield a unique solution for the particle absorption coefficient. Moteki et al. (2010) developed radiative transfer equations describing the filterbased measurements, taking into account the filter absorption and scattering optical depths, the asymmetry parameter, the filter geometry and material, and the particle penetration depth. In all cases, the models must be "adjusted" to fit to experimental observations.

An evaluation of many of the different correction algorithms has been carried out by comparing the model filter transmission functions with the transmittance measured from many experiments with different types of aerosols and particle sizes. The transmission function is the ratio of a reference absorption coefficient, $\sigma_{\text {ref }}$, to the attenuation, $\sigma_{\text {atn }}$, measured by a commonly implemented filter-based instrument, the Particle Soot Absorption Photometer (PSAP). In these experiments, the reference absorption coefficient was the collection of measurements with a photoacoustic spectrometer (described in Sect. 2.1.2), and the transmittance was the ratio of the light through the aerosol-laden filter to the light through the aerosol-free reference filter.

As seen in Fig. 1, the filter transmission function for the PSAP varies widely, depending on the type of aerosol and the transmission functions predicted by the various correction algorithms. The differences at this time have not been explained and from this figure we conclude that results from different calibration experiments can differ by up to $20 \%$ because of many partly resolved or unresolved problems that were listed above.

There have been many studies exploring instrument performance that highlighted the differences between 
instruments of the same or different type (cf. Müller et al., 2011). These studies, using various types of light absorbing materials, showed that the differences were often related to the type of material used; however, given that none of them met the criteria as SRM (see Sect. 3.1), it was difficult to interpret the results, i.e. to relate them to a specific characteristic of the materials that were used in the comparisons.

\subsubsection{Photoacoustic methods}

The photoacoustic measurement technique is based on the principle that particles that contain material whose internal energy is increased as they absorb photons will transfer that heat to the surrounding environment and raise the temperature (Petzold and Niessner, 1992; Arnott et al., 1999, 2000, 2003a, b, 2005; Arnott and Moosmüller, 2005; Lack et al., 2006). This principle is implemented by bringing the aerosols into a chamber and illuminating them with a oscillating laser. An acoustic wave, measured with a microphone, is generated by the increased temperature as the light absorbing particles heat the air. The amplitude of this wave is proportional to the absorption coefficient. As there are no intermediate filters involved with this technique, no corrections are needed for the artefacts introduced by the use of filters.

Unlike the filter-based technique for which no direct calibration method currently exists, the photoacoustic sensors can be directly calibrated with reference gases of known absorption cross sections. For example, photoacoustic photometers operating at $532 \mathrm{~nm}$, the wavelength at the ground where the solar radiance is approximately at a maximum, can be calibrated with either $\mathrm{NO}_{2}$ or $\mathrm{O}_{3}$. For longer wavelengths where reference gases are not available, a less accurate calibration method is used that measures the total extinction of the instrument's laser through the absorbing aerosol and subtracts from it the scattering coefficient measured separately with a nephelometer (Lewis et al., 2008).

Using the ozone calibration method, Lack et al. (2006) independently validated the photoacoustic technique for aerosol using size-selected spherical Nigrosin dye. This study showed that this technique can measure aerosol absorption to an accuracy of $<=5 \%$. This compares to varying estimates for filter-based absorption of 20-30\% accuracy. Lack et al. (2009) went on to validate the photoacoustic technique for measurement of the effects of internal mixing of an absorbing aerosol core. They showed that a photoacoustic spectrometer (PAS) can measure the enhanced absorption due to internal mixing to an accuracy of ca. $5 \%$, in addition to showing that the PSAP was not capable of measuring the same values. This study utilised calibrated (size and optical) absorbing PSL spheres. In addition to these validations, the PAS technique has been compared to the "difference method" for measuring absorption (extinction - scattering) and shows robust correlations (Virkkula et al., 2005).

"Given this validation of the PAS on constrained systems such as size-selected Nigrosin and mono-disperse absorb- ing polystyrene latex spheres (PSLs), in addition to the PAS performance against other methods of measuring absorption, the PAS can be rightfully set as a benchmark for measuring aerosol absorption." With this in mind, it has been suggested that further uncertainties in the filter-based absorption methods may exist, which are caused by organic aerosol coating the filter matrix, leading to absorption biases that are not accounted for in the standard filter-based absorption photometer corrections (Lack et al., 2008; Cappa et al., 2008).

\subsection{Challenges to rBC, EC and $\mathrm{OC}$ measurements}

Although there are a number of different techniques for measuring $\mathrm{rBC}, \mathrm{EC}, \mathrm{OC}$ and mixtures of these (soot), only the thermal optical analysis (TOA) and single particle incandescence techniques were discussed in depth. The TOA method evaluates mixtures of $\mathrm{OC}$ and $\mathrm{EC}$ and the incandescence technique looks at refractory black carbon (rBC), a form of carbon directly related to EC.

\subsubsection{Measurements of refractory black carbon}

The technique of continuous laser-induced incandescence has been implemented in the single particle soot photometer (SP2, Stephens et al., 2003; Schwarz et al., 2006) developed by Droplet Measurement Technologies (DMT). This technique retrieves the refractive black carbon (rBC) mass contained in a particle from the amount of visible light emitted when the particle is heated to $\sim 4200 \mathrm{~K}$ (Moteki and Kondo, 2010). Particles in the aerosol sample are aerodynamically directed into the center of a highly focused Nd:YAG laser beam $(\lambda=1064 \mathrm{~nm})$ with $100 \%$ collection efficiency. Particles containing at least $\sim 0.5 \mathrm{fg}$ of $\mathrm{rBC}$ will heat as they absorb the incident photons and vaporize, emitting thermal radiation in the visible. The light emitted by individual particles is collected by an incandescence detector, and its peak intensity is linearly proportional to the original $\mathrm{rBC}$ mass content of the particle over most of the mass range observed in the ambient. This relationship is largely independent of the morphology and independent of the mixing state of the rBC with other components (Moteki and Kondo, 2007; Slowik et al., 2007). In addition, light scattered by an individual particle, as it interacts with the laser, is collected and can provide information about the mixing state of the particle (Gao et al., 2007; Moteki and Kondo, 2007). The incandescence intensity to $\mathrm{rBC}$ mass relationship is established using various materials whose rBC mass is known (Schwarz et al., 2006).

All SP2s are built with the same optical geometry, detector and laser design; however, each SP2 has individual characteristics that must be determined by calibration, e.g. detector response, sensitivity settings affecting the detection range, time resolution, and operating laser intensity. In addition, individual users select their own calibration approach and analysis software but not all users are equally familiar with detection/interpretation issues. 
There are several calibration issues that the application of SRMs could address:

- The SP2 has a limited detection range in terms of rBC mass per particle, which has different implications than having limited sensitivity in terms of mass concentration.

- The calibration curve is dependent on the chemical structure of the calibration material (Kondo et al., 2009). The SP2, for example, is $\sim 23-29 \%$ less sensitive to fullerene soot than to Aquadag, which are two types of common calibration materials.

- There are uncertainties due to how the rBC mass of the calibration particle is selected, although the use of an aerosol particle mass analyzer (APM) will greatly reduce this uncertainty.

- Calibration using pure rBC obtained by thermodenuding ambient aerosol, coupled with an APM, depends on the removal of non-rBC coatings for mass determination, and thus raises questions about the effectiveness of such removal.

- There is an uncertainty in choosing the right calibration material for the sample studied, i.e. the calibration material should be representative of the environmental rBC being measured.

The SP2 measures $\mathrm{rBC}$ over a mass equivalent size range from approximately $80 \mathrm{~nm}$ to $900 \mathrm{~nm}$ (depending on the version of the SP2 used). In order to estimate the rBC mass outside the range of detection, the mass size distribution is fit with a $\log$ normal distribution. Since the majority of $\mathrm{rBC}$ mass in the environment is found in particles $>80 \mathrm{~nm}$, there is limited uncertainty in the estimate of mass below this size. However, if the ambient size distribution of $\mathrm{rBC}$ is nonlognormal, the uncertainty can be quite large when estimating the $\mathrm{rBC}$ mass in sizes larger than the detection limit.

\subsubsection{Measurements of EC and OC}

When measuring the separate OC and EC components of soot, we are faced with the fundamental problem that the distinction between OC and non-organic carbon is somewhat arbitrary (e.g. Seinfeld and Pandis, 1998). There seems to be no measurement technique that is able to rigorously determine the absolute amounts of EC and $\mathrm{OC}$ in a mixture of the two.

By definition, an atom should be regarded as EC only if imbedded in a large enough structure consisting EC only, independent of its allotropic form (diamond, graphite, amorphous). A few techniques can identify the nature of the atoms bonded to $\mathrm{C}$ in a material: ${ }^{13} \mathrm{C}$-Nuclear Magnetic Resonance (Casabianca et al., 2010), including Distortionless Enhancement by Polarization Transfer Spectra (Schmidt-Rohr and
Mao, 2002), and Near-Edge X-ray Absorption Fine Structure spectroscopy (Braun, 2005). However, C atoms can be well bonded to $\mathrm{C}$ atoms even in quite banal organic molecules like coronene or neopentane, for instance. Extended X-ray Absorption and Extended Energy Loss Fine Structure Spectroscopy are sensitive to the neighbors of the target atom up to 0.6 to $0.8 \mathrm{~nm}$ (e.g. Castrucci et al., 2009), which is still small for a nano-particle, and even for a PAH molecule (about 3 cycles' width). Calculated absorption spectra for large PAHs show that the strength of the $\mathrm{C}-\mathrm{C}$ bond still changes when shifting from $\mathrm{C}_{112} \mathrm{H}_{26}$ to $\mathrm{C}_{130} \mathrm{H}_{28}$ (Bauschlicher et al., 2008). In practice, although some of these techniques have been used for environmental analyses (e.g. apportioning the contribution of diesel exhaust and wood smoke in urban ambient PM, see Braun et al., 2008), they still are unable to distinguish amorphous or nanocrystalline EC from large PAHs.

While EC allotropes do not present any common spectroscopic peculiarity, all of the diamond, graphite, and amorphous carbons have a very low vapor pressure (actually one of the lowest among all elements on the Earth). Indeed, the enthalpy of sublimation is $>700 \mathrm{~kJ} \mathrm{~mol}^{-1}$ for graphite, as compared to 120 and $135 \mathrm{~kJ} \mathrm{~mol}^{-1}$ for levoglucosan and cellulose tar, respectively (CRC, 2011). The very low volatility of EC is actually the basis for most current analytical techniques which try to separate $\mathrm{OC}$ and $\mathrm{EC}$.

The mass of OC and EC in ambient particulate matter is typically derived with the thermal/optical analysis (TOA) technique. There are two types of TOA, off-line and semicontinuous. In the off-line technique, a punch from a filter sample is heated to high temperatures, either in an oxidizing atmosphere or first in an inert atmosphere followed by an oxidizing atmosphere. When OC associated with soot is heated in an inert atmosphere, some fraction of it chars into an EC-like, light-absorbing, refractory substance. TOA methods monitor either light transmission through (TOT; Birch and Cary, 1996) or reflection from (TOR; Chow et al., 1993) the sample punch to correct for the charred OC. The semi-continuous approach only differs in that there is a fixed filter in the system that captures the particles in realtime and is then subjected to the same temperature cycles as in the off-line technique (Johnson et al., 1981; Turpin et al., 1990). With this technique the OC/EC concentrations are determined approximately every ten minutes, depending on the mass concentrations in the environment. Figure 2, taken from Subramanian et al. (2006) with the permission of the journal Aerosol Science and Technology, illustrates how this technique is implemented, as well as demonstrating one of the uncertainties inherent in this method. Two typical thermograms from the analysis of a filter punch using the He4-870 and He4-700 protocols are shown in top and bottom panels of this figure, respectively. The formation of light-absorbing pyrolyzed carbon (PC) causes the transmission of laser light through the sample (and filter) to decrease through much of the He-mode of the analysis. The transmission reaches 


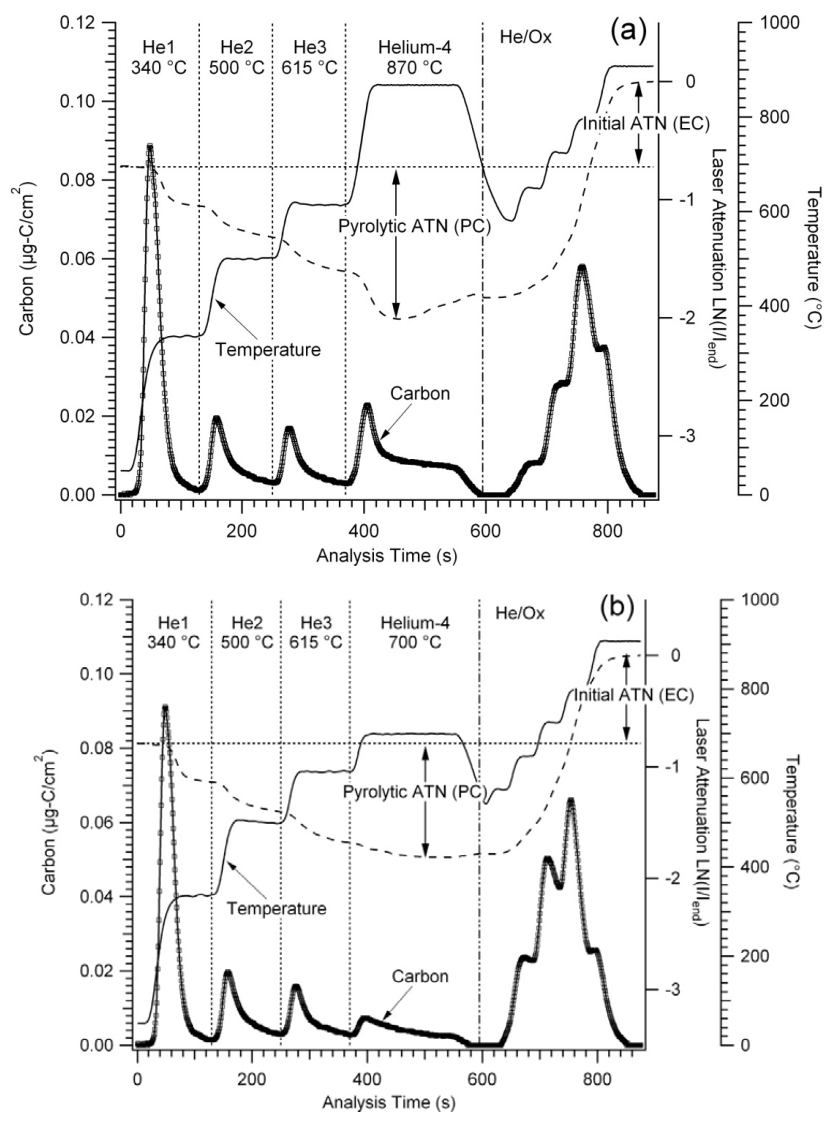

Fig. 2. Thermograms from the analysis of a typical ambient sample with the (a) He4-870 and (b) He4-700 protocols. The thermograms are for parallel punches of the same filter. Dashed line indicates laser attenuation. There is clear loss of light-absorbing carbon in the He-mode of the He4-870 protocol but not in the He-mode of the He4-700 protocol (Subramanian et al., 2006).

a minimum value partway through the $870{ }^{\circ} \mathrm{C}$ temperature step of the He-mode (top panel) and then begins increasing, indicating premature evolution of EC. The bottom panel of Fig. 2 shows a thermogram from the He4-700 analysis of a second punch from the same filter shown in the upper panel of Fig. 2. The laser signal reaches its minimum value in the $\mathrm{He} 4$ step $\left(700^{\circ} \mathrm{C}\right)$ and does not increase until the $\mathrm{He} / \mathrm{Ox}$ mode suggesting no loss of EC in the He-mode. This does not guarantee that $\mathrm{EC}$ is not lost in the He-mode because the loss of EC could be offset by continued pyrolysis.

The relationship between the derived EC and the temperature protocol has been studied by a number of investigators (e.g. Chow et al., 2001, 2004; Yang and Yu, 2002; Conny et al., 2003; Schauer et al., 2003). Decreasing the temperatures of the stages in the He-mode can increase the measured EC due to OC not completely evolving or not pyrolyzing into light-absorbing carbon (Conny et al., 2003; Schauer et al., 2003). When the peak inert-mode temperature is set too high, this can also bias the OC/EC split due to premature EC evolution (Chow et al., 2001). The effect on the derived
$\mathrm{OC} / \mathrm{EC}$ as a result of different temperature protocols is one of several factors that contribute to the uncertainty in measurements using these TOA techniques (other uncertainty factors are discussed further on in this section). The standard procedure, recommended by the manufacturers of commercial TOA systems, for calibrating the TOA is to prepare preconditioned filters with organic material, like sucrose or potassium hydrogen phthalate (KHP), whose number of carbon atoms is precisely known.

These are then analyzed, providing an accurate procedure for relating the output of the analyzer of carbon dioxide or methane (depending on the TOA technique used) with the number of carbon atoms on the filter. This does not, however, test the fidelity of the technique for separating the $\mathrm{EC}$ from the OC since the carbon from these calibration materials contains no EC. Calibration may also be carried out by injection of $\mathrm{CO}_{2}$ directly into the combustion oven. the Sunset Lab. instruments always inject a calibration amount of $\mathrm{CH} 4$ at the end of each analysis cycle. This is a standard procedure for certain semi-continuous analyzers, which may also be implemented for offline TOA instruments (Cavalli et al., 2010).

Intercomparisons between the TOA methods have shown that the temperature protocol and the optical detection technique (transmission versus reflection) can make a significant difference in the measured EC content (Chow et al., 2001; Schmid et al., 2001; Schauer et al., 2003; Subramanian et al., 2006; Cavalli et al., 2010; Piazzalunga et al., 2011). Determining the temperature at which all OC and no EC evolves in $\mathrm{He}$, air or $\mathrm{O}_{2}$ is challenging. It may be possible to determine the temperature below which no known form of pure EC from any source evolves at a significant rate (still to be determined). But this does not guarantee that organic molecules will evolve only below this temperature and, indeed, not all organic matter chars or evolves at a lower temperature than pure EC (Schauer et al., 2003).

"Low temperature protocols have been shown to overestimate EC in the presence of resilient organics, such as those recently detected by Piazzalunga et al. (2011) in aerosol samples collected at a heavily polluted site. On the other hand, as previously noted, high temperature protocols can lead to the premature evolution of EC." Inorganic matter such as mineral oxides and salts, found in biomass smoke or sea spray (such as $\mathrm{KCl}$ or $\mathrm{NaCl}$ ), can also alter the temperature at which complex/large organic compounds and EC evolve off the filter (Novakov and Corrigan, 1995; Boparai et al., 2008; Wang et al., 2010). Hence, the temperature split point between OC and EC remains arbitrary.

The removal of water-soluble organic compounds from the filter by washing procedures significantly reduces pyrolysis during the thermal analysis (Piazzalunga et al., 2011). Furthermore, the washing procedure also removes other soluble components in the sample (i.e. inorganic salts like $\mathrm{KCl}$ ) that may reduce the EC oxidation temperature. The application of thermal protocols characterized by long enough time steps at low temperature allows a complete evolution of the sample, 
leading to a significant reduction in $\mathrm{PC}$ formation both in washed and untreated samples (Chow et al., 1993; Piazzalunga et al., 2011).

Another potentially important interference during TOA determination of soot is the presence of carbonate carbon (CC). This form of inorganic, refractory carbon produces an additional $\mathrm{CO}_{2}$ signal and hence inferred carbon mass in atmospheric aerosol samples. Pure EC-OC RM may not provide adequate simulation of atmospheric samples with significant CC content. $\mathrm{CC}$ is present in natural ground and building/demolition dust and is mostly related with coarse aerosol. Its contribution to total particulate carbon concentration is generally considered negligible but this may not be the case in certain areas, such as Southern Europe and across the Mediterranean basin, where soil dust outbreaks are frequent and greatly increase the ambient PM air levels (Karanasiou et al., 2011; Robles et al., 2011). If CC is not taken into account, concentrations of OC or EC may be overestimated, depending on the thermal protocol and position of the split point. The problem of a potential interference of $\mathrm{CC}$ with the signal of EC or OC has been mentioned, but not assessed, in several EC/OC inter-comparison studies (Jankowski et al., 2008). Recent research has also demonstrated that the presence of metal oxides/carbonate mixture may lower the decomposition temperature in thermal/optical carbon analysis (Robles et al., 2011).

Determining the accuracy of different TOA techniques with ambient or atmospherically-relevant samples is difficult, however, since no technique can unambiguously determine the true value for the actual chemical composition - the fraction of OC/EC - of the sample. Hence, to assess the accuracy of TOA techniques, reference materials for EC are necessary. Any material consisting of $100 \% \mathrm{EC}$ according to an elemental analysis (i.e. no $\mathrm{H}$, no $\mathrm{O}$, no $\mathrm{N}$ ) could a priori be regarded as a suitable reference material; however, the best standard for atmospheric EC determination would be the most reactive (i.e. least refractory) material among all kinds of soot produced by various techniques as long as it is pure EC. Ideally, a standard reference material should also be produced as a mixture of this EC with non-volatile organics prone to charring in thermal analysis, like e.g. fulvic acids. For estimating soot reactivity in thermal oxidation processes, Schmid et al. (2011) established a relative reactivity index, based on one hand on calibration with an inert graphite, and on the other hand by a completely distorted (and hence enormously reactive) spark-discharge soot. This represents the range of achievable soot reactivity, and has become a valuable tool for the engine developers, who are eager to get highest soot reactivity for effective after-treatment.

\section{Soot reference materials}

The common theme, highlighted when summarizing the primary obstacles for improving the accuracy and reducing the uncertainties measurements of soot properties, was the need for SRMs for calibrating, validating and comparing instruments. The following sections present the various dimensions of this issue.

\subsection{Defining the meaning of a reference material}

According to the ISO Guide (2008), provided by the International Organization for Standardization, reference materials (RM) are defined as:

Material, sufficiently homogeneous and stable with respect to one or more specified properties, which has been established to be fit for its intended use in a measurement process.

The uses of RM may include the calibration of a measurement system, assessment of a measurement procedure, assigning values to other materials, and quality control; however, a single RM should not be used for both calibration and validation of results in the same measurement procedure. Calibration RMs, for example, are typically used to establish scaling constants and determine the linearity of the various components of a measurement system, whereas validation RMs are needed to test the complete measurement system, preferably with material that represents the natural environment. The RM is characterized by a metrologically valid procedure for one or more specified properties, accompanied by a certificate that provides the value of the specified property, its associated uncertainty, and a statement of metrological traceability. Uncertainties for such attributes may be expressed as probabilities. Metrologically valid procedures for the production and certification of reference materials are given in, among others, ISO Guide (2006, 2009).

The ISO Guide defines "certified" reference materials (CRM) as

Reference material characterized by a metrologically valid procedure for one or more specified properties, accompanied by a certificate that provides the value of the specified property, its associated uncertainty, and a statement of metrological traceability.

In the United States, the National Institute of Standards and Technology (NIST), previously the National Bureau of Standards, maintains many RMs and CRMs. There are a number of modes for assigning values to RMs for chemical composition. These take the form of certified value $(\mathrm{C})$, reference value only $(\mathrm{R})$ or information only $(\mathrm{I})$. These modes are:

- Certification at NIST using a primary method with confirmation by other method(s) (C)

- Certification at NIST using two independent criticallyevaluated methods $(\mathrm{C}, \mathrm{R})$

- Certification/value-assignment using one method at NIST and different methods by outside collaborating laboratories $(\mathrm{C}, \mathrm{R})$ 
- Value-assignment based on measurements by two or more laboratories using different methods in collaboration with NIST (R, I)

- Value-assignment based on a method-specific protocol $(\mathrm{R}, \mathrm{I})$

- Value-assignment based on NIST measurements using a single method or measurements by an outside collaborating laboratory using a single method $(\mathrm{R}, \mathrm{I})$

- Value-assignment based on selected data from interlaboratory studies (R, I)

The concept of the "Independent Methods" has been developed at NIST using the following guidelines:

- One criterion for a standard sample is "Its composition should have been determined by independent and reliable methods affording agreeing results" (Hillebrand, 1916)

- Modes of establishing the accuracy of National Bureau of Standards (NBS) RMs defined as "reference method, two independent methods, or interlaboratory comparison" (Yolken, 1973)

- Three modes of measurement are used to assure that the values of the RM property(ies) are accurate. These are (a) definitive methods, (b) reference methods, and (c) two or more independent and reliable methods. (Cali and Reed, 1976). Adapted from Epstein (1991).

\subsection{Potential soot reference materials}

\subsubsection{Currently implemented material}

At present there are no materials that have been shown to meet either the ISO or NIST definitions for RM or CRM for soot properties. There are, however, numerous techniques for producing soot particles that might meet the requirements for an SRM. Some of these use methods to continuously produce soot particles while others are fabricated, put in a liquid suspension and bottled for application on filters or nebulization. Of the continuous type, those frequently used are:

- Laser breakdown of acetylene (Spanner et al., 1994)

- Soot recondensation from carbon filament (Niessner and Helsper, 1985)

- Laser ablation from solid graphite

- Aerosolization of pyrolyzable material

- Flame-derived soot production (Kirchstetter and Novakov, 2007).

- Combusted kerosene soot (Popovicheva et al., 2003).
- Spark discharge soot (Helsper et al., 1993)

The fabricated particles, for example, are:

- Industrial carbon blacks (used in printing, plastics, coatings, e.g. Monarch 71, Printex \#, Tokai Aqua Black 162)

- Absorbing polystyrene latex (PSL) spheres

- Absorbing glassy carbon spheres

- Nigrosin (synthetic dye)

- Aquadag (aqueous-based colloidal dispersion of ultrafine graphite)

- Fullerene soot

- Tailored graphitized thermal soot (Popovicheva et al., 2011)

Note that many of these types of particles are not soot, per se, because they are not produced from combustion; however, they have some characteristics of soot that can be used to exercise a sensor in an instrument.

Within the context of how RM is defined in the ISO guide, we are looking for soot-like materials sufficiently homogeneous and stable with respect to one or more specified properties, which have been established to be fit for their intended use in a measurement process. This requires first defining the property of soot that we wish to measure, then assessing if a candidate RM is fit for its intended use. At the moment, the most frequently desired properties of airborne soot are (1) optical (scattering and absorption coefficients and asymmetry parameter), (2) physical (size distribution of number and mass concentrations), (3) chemical (mass of OC and EC, and (4) cloud forming potential, i.e. cloud condensation and ice nuclei ( $\mathrm{CCN}$ and IN) fraction.

None of the potential RM listed above have been established to be fit for its intended use in a measurement process, i.e. each of the materials has been used in one or more applications to test the operation of a measurement system and, in some cases, to even calibrate instruments; however, the community of instrument users have not reached a consensus regarding either the soot material (or proxy) or the method to apply them.

Hence, within the context of the soot properties and measurement techniques discussed during the workshop, SRMs are needed for filter-based light transmission and photoacoustic techniques to measure light absorption, for laserinduced incandescence for $\mathrm{rBC}$ measurements and for TOA that derives OC and EC concentrations. There are some commonalties amongst the different techniques that could allow the same SRM to be used for comparing different instruments, e.g. the filter-based light transmission and TOA measurements both involve the use of aerosol laden filters; hence, pre-prepared filters with SRMs of known optical properties 


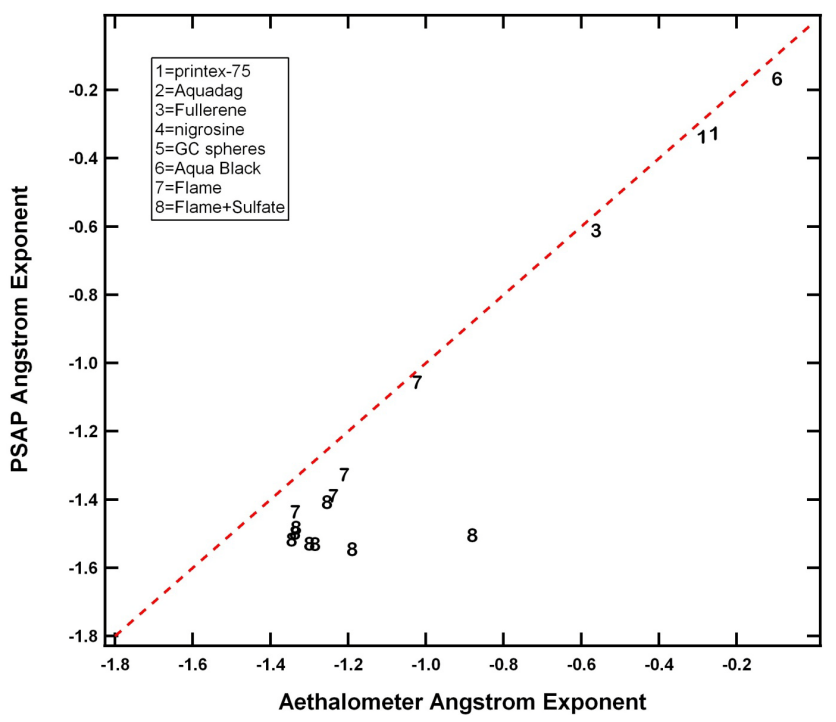

Fig. 3. The angstrom coefficients calculated from a multiwavelength PSAP and Aethalometer measurements of different types of light absorbing particles are compared in this figure.

and $\mathrm{OC} / \mathrm{EC}$ concentrations could be used in both types of instruments. Similarly, the SP2 and photoacoustic instruments measure the aerosols suspended in air passing through their sample chambers. If an SRM can be generated with known light absorption cross section and mass concentration of $\mathrm{rBC}$, then it would be a useful RM for comparing the two types of instruments, as well as calibrating and evaluating them under different conditions.

In the following four sections the individual SRM needs of the different measurement techniques are discussed, with mention of potential overlapping requirements, as appropriate.

\subsubsection{SRM for filter-based light transmission techniques}

At the moment, none of the filter-based techniques are currently being calibrated or compared using SRMs whose characteristics are well defined with respect to their optical properties. During the EUSAAR2009 experiments (Müller et al., 2011), the responses of several Aethalometers and PSAPs were evaluated against test aerosol including absorbing PSL spheres. Absorbing PSL has many features of an attractive RM that has discrete sizes and whose optical properties are known. In these experiments, it was observed that the PSL type used, although non-absorbing at $781 \mathrm{~nm}$, could be used for other regions of the spectral range. The correction algorithms applied for the conversion of the attenuation signal to absorption coefficient gave consistent results but with significant variability, up to $30 \%$, compared to the photoacoustic reference instruments. These types of particles, however, do not represent atmospheric soot. They may be convenient for some types of comparisons or calibrations but they do not ad-
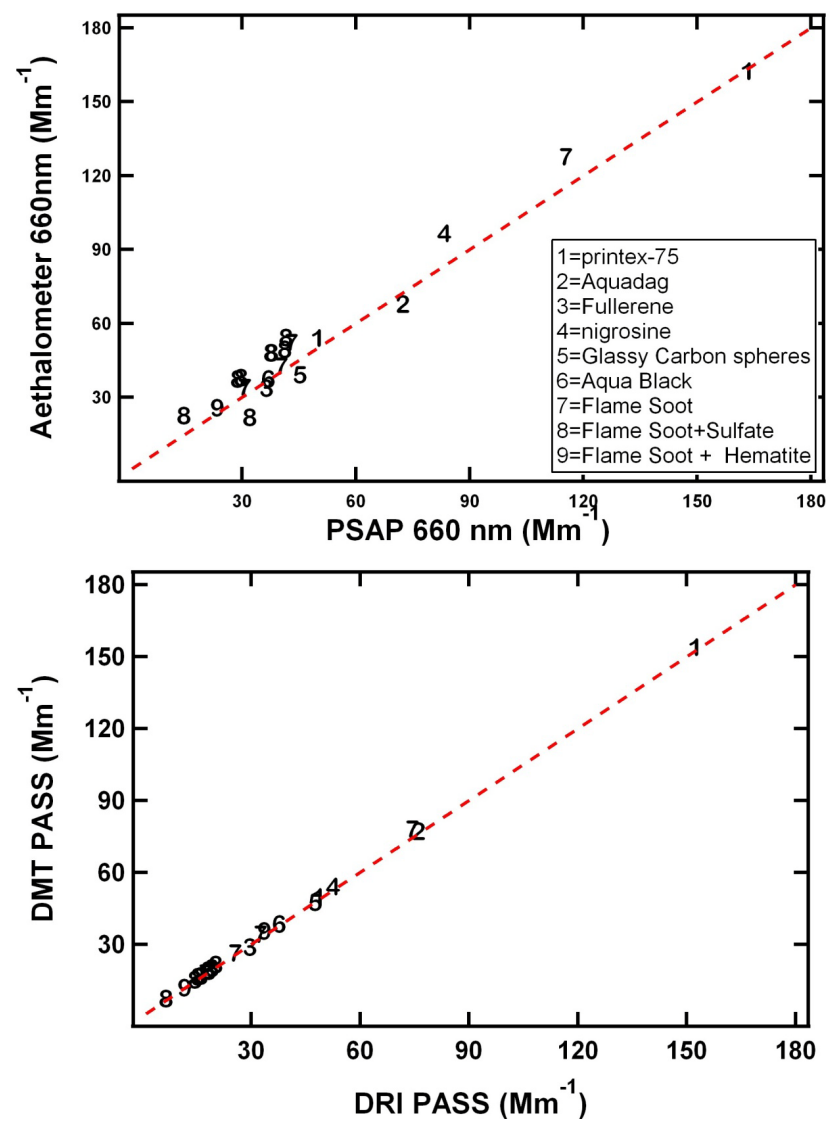

Fig. 4. The absorption coefficients measured with a PSAP and Aethalometer are compared for different types of light absorbing particles in the top panel. In the bottom panel, two photoacoustic absorption spectrometers are compared with the same set of materials.

dress the problems that arise due to the more complex mixtures of EC, OC, dust and inorganics found in the natural environment.

As previously shown in Fig. 1, conducting laboratory evaluations with various light absorbing aerosols indicates that the correction algorithms do not account for the differences in properties of the various types of light absorbing materials. This highlights the value of using soot SRMs whose optical properties are well characterized, as well as their physical and chemical properties, to refine the current suite of models that are being used to remove the measurement artifacts. Figures 3 and 4 help further illustrate the value of SRMs to evaluate how optical properties are measured by different techniques. In a study called the Boulder Light Absorbing Carbon (BLAC) experiment, several photoacoustic and filter-based instruments were challenged with 10 different types of light absorbing aerosol particles. Figure 3 shows a comparison of the Angström exponent measured with a multi-wavelength Aethalometer and PSAP. More information is available by contacting Patrick Sheridan (patrick.sheridan@noaa.gov). 
As seen in this figure, there is relatively good agreement between the derived exponents for Printex-75, Fullerene, Aqua Black and flame soot (Kirchstetter and Novakov, 2007). When the flame soot is mixed with various fractions of ammonium sulfate, however, a large amount of variation is seen, with the PSAP remaining constant while the Aethalometer varies by more than $50 \%$. Although corrections had been applied to the PSAP and Aethalometer measurements, no efforts have been made at this time to evaluate these discrepancies by applying the various models that are currently being debated in this community of users.

Figure 4 (bottom panel) shows comparisons of the absorption coefficient measured by two PAS units, one built at the Desert Research Institute (DRI) and the other, using a similar design, built by Droplet Measurement Technologies. This comparison shows that the agreement is quite good over the entire range of light absorbing materials. In the top panel of Fig. 4 is a similar comparison, using the same range of particle types, between an Aethalometer and PSAP at the same wavelength. The agreement between the two filterbased techniques is reasonably good, but as with the comparison of the Angström exponents, introducing the ammonium sulfate mixed with flame soot increases the variability.

At this time, it is not clear if any of the aforementioned materials listed in Sect. 3.2.1, or the light absorbing particles used in the comparisons shown in Figs. 3 and 4, can serve as SRM for filter-based light absorption measurement techniques since not all of them have had their optical properties sufficiently characterized. Compounding the problem is that, to date, no methods have been developed that can be used to apply a known, repeatable amount of SRM to the filters used in these techniques. One of the challenges for implementing a soot SRM, once appropriate materials are selected, is the application of the material to the instrument since instruments like the Aethalometer measure changes in the attenuation as the aerosol particles accumulate on the filter. Hence, the SRM cannot just be weighed and deposited on the filter, but will possibly need some type of delivery system that provides the SRM at a known concentration rate.

\subsubsection{RM for photoacoustic light absorption techniques}

The users of the PAS technique can use the same SRMs that are developed for evaluating filter-based techniques, as was illustrated above in the examples shown in Figs. 3 and 4. Although those particular examples suggest that the PAS technique does not appear to be sensitive to the composition of the particles, this specific test was carried out at only a single wavelength of $870 \mathrm{~nm}$. A much broader evaluation is needed at shorter wavelengths to assess the sensitivity of the PAS technique to materials like dust and some types of OC produced by wood burning that are known to absorb in the ultraviolet wavelengths.

\subsubsection{RM for laser-induced incandescence techniques}

The majority of SP2 users belong to an active working group that discusses issues related to SRMs for calibration and comparison and methodologies for administering the SRMs. At present there are four materials commonly used to absolutely calibrate SP2: glassy carbon spheres, fullerene soot, Aquadag and thermally denuded ambient rBC.

\subsubsection{RM for TOA techniques}

A number of potential SRM candidates for TOA have been introduced in recent years (Yang and Yu, 2002; Chen et al., 2004; Klouda et al., 2005); however, none have been universally accepted as a standard by those who measure the OC/EC component of soot. For example, a filter-based NIST standard, reference material 8785 , was specifically developed with the intent of calibrating TOA methods. This RM is produced by resuspending urban dust (NIST standard 1649a) and filtering it with quartz fiber filters (Klouda et al., 2005). These filters were evaluated with a number of standard methodologies - the Interagency Monitoring of Protected Visual Environments (IMPROVE) and Speciation Trends Network-National Institute of Occupational Safety and Health (STN-NIOSH) protocols (Chow et al., 1993; Birch and Cary, 1996; NIOSH, 1999) - with mixed results. The total carbon, i.e. the sum of the EC and OC, agreed quite well; however, the measured fraction of OC differed by more than $60 \%$ (Klouda et al., 2005). The source of the differences between the two techniques was not resolved other than concluding that the separation of EC and OC is methoddependent with no way to assess which of the two protocols was providing the "correct" value.

There are a number of materials, listed in Sect. 3.2.1, that are currently being used by various researchers. One of these that has been well documented (Stipte et al., 2005; Kirchstetter and Novakov, 2007) is diffusion flame soot, produced by burning methane in an inverted flow reactor. It has two reported advantages that make it a potential SRM: (1) the particle generation is stable and repeatable in size and concentration, and (2) the stability of the flame facilitates collection of the particles on a filter. The CAST soot generator (http://www.sootgenerator.com/) also uses a well defined diffusion flame from either gaseous or liquid fuel and has the same advantages. Although these characteristics have been reported in the open literature, there remains the need for much more extensive studies that substantiate the properties that would confirm that the diffusion flame soot could be used as an SRM.

Another source of reference particles that has been standardized for use in calibrating the German VDI/DIN extraction and thermal desorption technique for measuring soot is the Palas GFG spark discharge aerosol generator (Roth et al., 2004). As advertized, it produces a constant, reproducible particle size and concentration and has been widely used for 
studies of soot microstructure, composition, reactivity and oxidation. It is not, however, a combustion aerosol. It is used, in context with the comparison to an inert graphitic soot, to represent the full range of oxidative reactivity (Schmid et al., 2011).

A recent development (Popovicheva et al., 2011) is the creation of tailored soot whereby graphitized thermal soot is precisely coated with a known amount of organic carbon. With this approach, the EC/OC ratio is precisely controlled such that TOA techniques can be evaluated with respect to temperature protocols, repeatability, etc. Given that previous round-robin tests and intercomparisons of different techniques using urban dust or ambient samples experience the largest discrepancies when evaluating the EC/OC split, a tailored reference material where this ratio is well-known has a distinct advantage. It should be noted, however, that one requirement from the aerosol community that was openly stated is that the EC and the OC/EC ratio of such a material should resemble the $\mathrm{EC}$ and the OC/EC ratio of ambient aerosol, as should its refractivity. The tailored materials do not as yet meet this requirement because the $\mathrm{EC}$ in these materials evolves at a much higher temperature $\left(>800^{\circ} \mathrm{C}\right)$ than ambient EC. Work is currently underway to produce a tailored RM with EC that evolves at a lower temperature.

Any of the above sources of soot have the potential for serving as SRM for use with TOA; however, they must be homogeneously deposited on clean filters with a well-known mass within the appropriate concentration range for each instrument to avoid saturation. The tailored soot, i.e. OCcoated graphitized thermal soot, can be placed directly in the oven of the TOA instrument, on a clean quartz substrate, thereby avoiding issues associated with contaminated filters.

It is important that the method of application, i.e. how the RM is introduced to the measurement system, does not affect the response of the TOA to the SRM and that the method of application is a part of the SRM protocol. In addition, if the $\mathrm{EC} / \mathrm{OC}$ fraction is to be determined, then this parameter must be fully characterized for the selected SRM and remain constant with time.

\section{Recommendations}

During the course of the working group discussions, numerous suggestions were put forward by the participants related to how the problems associated with the lack of SRMs might be resolved. In general, irrespective of the measurement technique, SRMs are needed for addressing several pervasive problems:

- Calibrating sensors with environmentally relevant particles.

- Understanding measurement artifacts.

- Comparing data sets taken with similar or different techniques.
The recommendations that evolved from the discussions were related to resolving these problems and fall into two general categories: (1) SRM materials and application methodologies, and (2) user and manufacturer responsibilities. We have tried to consolidate the many suggestions and ideas that evolved during the meeting into a concise few that can most easily be implemented with application of realistic resources.

\subsection{SRM materials and application methodologies}

\subsubsection{Light absorption techniques}

Recommendation for calibrating filter-based light absorption instruments: a reference material with a well defined light absorption cross section and a methodology for using it should be developed.

At the moment there are no procedures to calibrate filterbased instruments that use the measurement of light attenuation to derive the absorption coefficient. Ideally, the approach would generate the calibration particles with known size and concentration to be sampled by the instruments with their normal inlet system. Given the many issues related to artifacts due to light scattering, humidity, loading, etc., the generated particles or the reference filter with the deposited particles will need to be carefully characterized with respect to the optical properties and the repeatability. The primary purpose is to provide a way to verify that the instrument is operating within the expected uncertainties.

Recommendation for understanding measurement artifacts in filter-based light absorption instruments: a set of reference materials with well known properties, and a methodology for using them, should be developed.

The light absorption coefficient is derived from the measurement of light attenuation through the use of various optical models that take into account the various interferences that produce artifacts and bias the results. Given that these artifacts are sensitive to the properties of the soot aerosols, i.e. size distribution, scattering coefficient and chemical composition, models need to be evaluated with a wide variety of reference materials that have had these properties completely characterized. The particles should be representative of relevant aerosol types, e.g. ambient aerosols and combustion particles. A broad range of relevant aerosol optical properties, e.g. single scattering albedo, scattering and absorption, Angström exponents and extinction, should be covered by the aerosols used in the model evaluations.

Recommendation for evaluating and comparing instruments: Design and conduct an international experiment to compare and evaluate techniques for measuring light absorption.

A well designed study to compare and evaluate measurement techniques is needed once reference materials have been found that are suitable for evaluating the artifacts, as described in the previous recommendation. As previously 


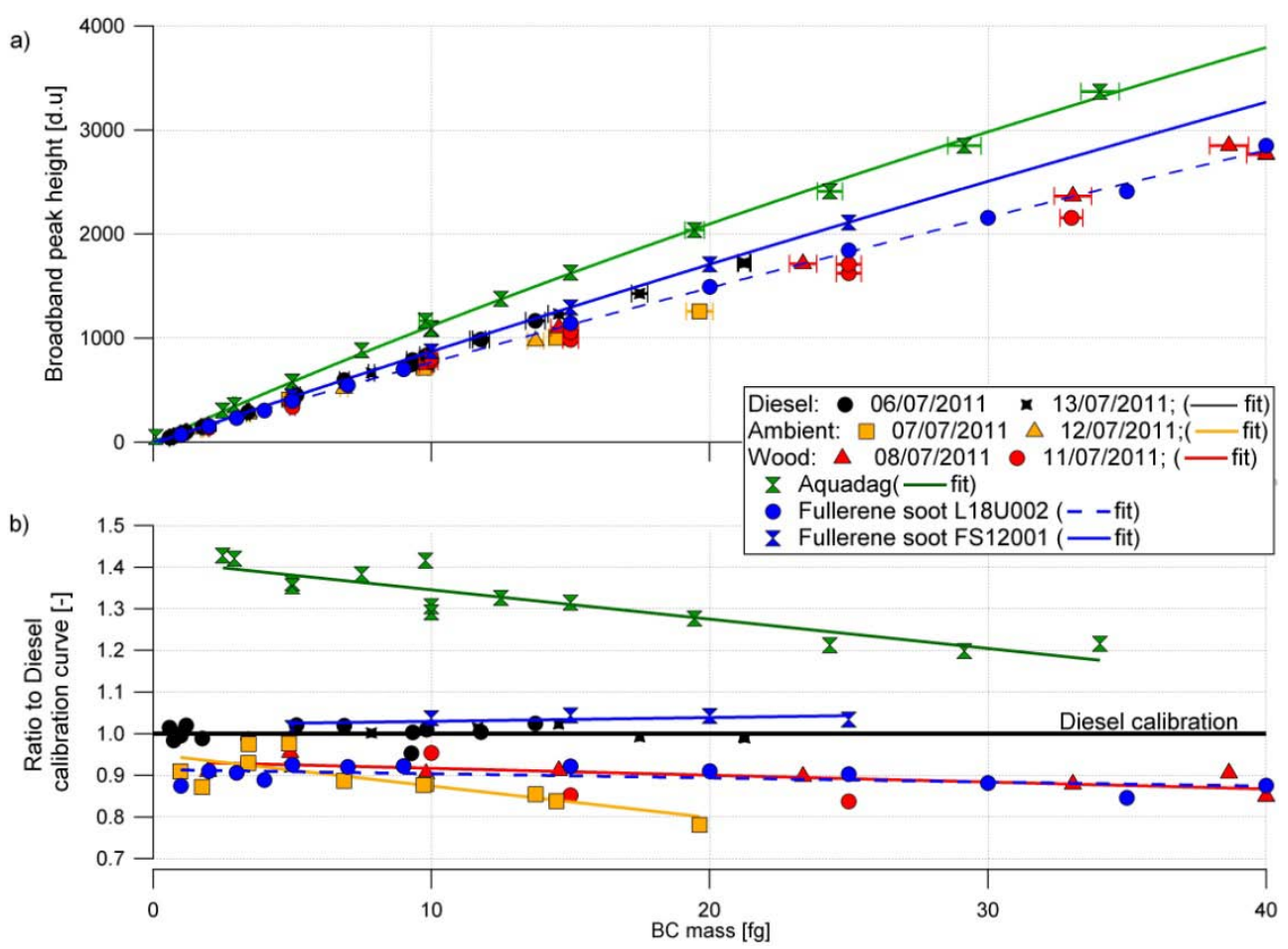

Fig. 5. This figure, taken from Laborde et al. (2012a) illustrates the response of an SP2 to a range of reference materials and ambient particles.

discussed, a number of valuable laboratory studies have been conducted that evaluated instrument performance of selected types of instruments; however, none of these studies were done using reference materials with well characterized properties. The recommended study would include as many of the different techniques as possible, i.e. filter-based, photoacoustic, cavity ring-down, etc., with more than one instrument representing each technique. This type of study, using SRMs with known properties, would provide a balanced, unbiased evaluation that would assess the respective strengths and weaknesses of the different techniques and help estimate the uncertainties as a function of the SRM properties. In addition, it would help to identify the best optical model for correcting the artifacts in filter-based methods.

\subsubsection{Refractory black carbon measurements}

Recommendation for calibrating and comparing single particle laser incandescence techniques: a centralized source of well characterized fullerene soot is needed.

The community of SP2 users, in concurrence with recent publications (Moteki and Kondo, 2010; Gysel et al., 2011; Laborde, et al., 2012a), have identified fullerene soot as the best SRM for calibrating and comparing the SP2s because of its similarity to ambient refractory black carbon (Fig. 5). Calibration particles are ideally selected by mass using an APM or a centrifugal particle mass analyzer (CPMA). However, these instruments are not widely available to most SP2 users. Hence, the established calibration method is to use a differential mobility analyzer (DMA) to select the particles by their mobility diameter. This requires knowledge of the calibration materials effective density (mass - mobility relationship), which may vary from batch to batch (Laborde et al., 2012a). Therefore a central source of well characterized fullerene soot is needed to assure reproducibility and accuracy of SP2 measurements by different research groups. The developer and vendor of the SP2, Droplet Measurement Technologies, is in the best position to act as this source.

An alternative option is needed for SP2 calibrations performed in the interim until the central source of fullerene soot is available, as well as to allow previous SP2 measurements to be related. Aquadag based SP2 broadband incandescence calibration curves can be recalculated to fullerene soot equivalent SP2 calibration, assuming a linear calibration with axis intercept at zero, using the following approach:

$s_{\mathrm{FSeq}}\left(m_{\mathrm{BC}}\right)=\frac{s_{\mathrm{AQ}}\left(m_{\mathrm{AQ}}\right)}{m}{ }_{\mathrm{AQ}} r_{\mathrm{FS} 2 \mathrm{AQ}} m_{\mathrm{BC}}$.

$s_{\mathrm{FSeq}}$ is the fullerene soot equivalent SP2 signal amplitude as a function of $\mathrm{BC}$ mass $\left(m_{\mathrm{BC}}\right)$, i.e. the fullerene soot equivalent calibration curve, $s_{\mathrm{AQ}}$ is the measured SP2 incandescence signal amplitude for Aquadag as a function of Aquadag mass $\left(m_{\mathrm{AQ}}\right)$, i.e. the Aquadag calibration curve, and $r_{\mathrm{FS} 2 \mathrm{AQ}}$ is the ratio between the SP2's signal amplitudes for fullerene soot and Aquadag particles of equal mass. The relative sensitivity of the SP2 to fullerene soot and Aquadag has been shown to be almost equal for 9 different SP2 


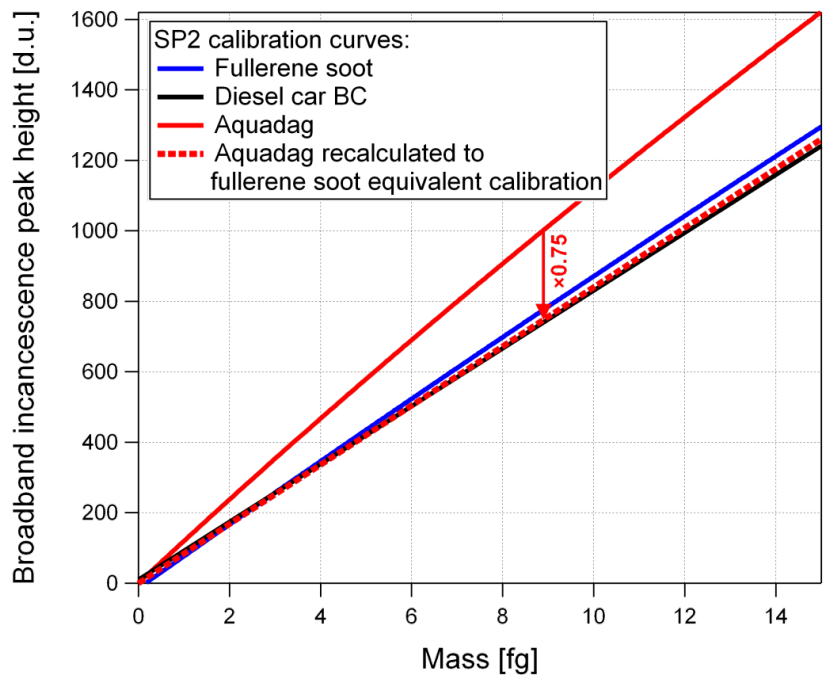

Fig. 6. Calibration curves of an SP2 for fullerene soot (blue line), diesel car BC (black line) and Aquadag (solid red line) from Laborde et al. (2012a). The red line shows a fullerene soot equivalent calibration obtained by recalculating the Aquadag calibration using Eq. (1) and a correction factor of $\sim r_{\mathrm{FS} 2 \mathrm{AQ}}=0.75$, as determined at a $\mathrm{BC}$ mass of $8.9 \mathrm{fg}$ in an intercomparison of $9 \mathrm{SP} 2$ instruments (Laborde et al., 2012b).

instruments, with an average value of $r_{\mathrm{FS} 2 \mathrm{AQ}}=0.75$ at a BC mass of $8.9 \mathrm{fg}$ (Laborde et al., 2012b). Consequently, the fullerene soot equivalent SP2 calibration should be derived from the Aquadag calibration curve $s_{\mathrm{AQ}}$ at an Aquadag mass near $m_{\mathrm{AQ}}=8.9 \mathrm{fg}$, as done in Fig. 6. This particular mass was chosen in the range where ambient $\mathrm{BC}$ mass size distributions typically peak. This is valid for SP2s of the current generation, which use a H6779 photomultiplier tube (Hamamatsu Inc., Japan) behind a Schott KG-5 filter to measure broadband incandescence signals. Hence, in general practice until more complete calibrations can be carried out, a single aquadag calibration at mobility diameter $300 \mathrm{~nm}(8.9 \mathrm{fg}$ aquadag mass) is sufficient to determine the broadband SP2 sensitivity to ambient $\mathrm{rBC}$ after application of the 0.75 factor to the modal peak height.

\subsubsection{Organic and elemental carbon measurements}

Recommendation for calibrating TOA instruments: the community of TOA users must agree on the best reference materials for calibrating the system response to $O C, E C$ and mixtures of $O C$ and $E C$.

The largest uncertainties and limitations when measuring OC and EC with the TOA technique are due to artifacts introduced by the pyrolysis (charring) of OC into EC. Various protocols have been implemented to minimize these artifacts but to date none have been universally accepted, largely because of the complexities related to the determination of the "true" OC and EC values and the validation of the optimum protocol.
The corrections that are applied in TOA to account for these artifacts rely upon measurements of the transmission through or reflectance of light from the aerosol loaded filter as it is heated to determine when charring occurs. There can be significant uncertainties associated with this correction due to differences in the specific mass absorption of native EC and the formed char, as well as the temperature protocol that is used, as was demonstrated in the example shown in Fig. 2 (Subramanian et al., 2006). These uncertainties must be accurately identified and quantified with SRMs with well known properties.

Given that the calibration procedure of TOA systems, at this time, is only for $\mathrm{OC}$, a reference material is needed for calibrating these systems over the entire temperature range so that EC is included. A number of candidates for this material have been identified, in particular flame generated soot or tailored graphitic thermal soot; however, the workshop ended with no consensus with respect to selecting an SRM. Once the EC material is agreed upon, the response of the TOA to mixtures containing refractory and charring OC can be evaluated. Additional, intensive campaigns are needed to demonstrate the advantages and disadvantages of any SRM.

Recommendation for understanding measurement artifacts in TOA systems: a set of reference materials with well known properties, and a methodology for using them, should be developed.

SRMs that contain chemical compounds other than OC and EC are needed to evaluate the artifacts that are introduced by the presence of these compounds. In addition to uncertainties related to charring during the derivation of $\mathrm{OC}$ and $\mathrm{EC}$ in soot, when various salts or metal oxides are present, there can be a catalytic degradation of the EC during the temperature stage when $\mathrm{OC}$ evolves. This is an additional artifact that produces a positive bias in the derived OC (i.e. Novakov and Corrigan, 1995; Yu et al., 2002; Subramanian et al., 2006; Cavalli et al., 2010; Hitzenberger and Rosati, 2010; Bladt et al., 2012). Once the SRM has been selected for the calibration of OC and EC response and evaluation of protocols to reduce charring, known quantities of various salts, metal oxides, refractive organic compounds and fulvic acids can be introduced to better quantify the relationship between $\mathrm{EC}$ degradation and the presence of non-OC/EC material concentrations.

As with the use of SRM mixtures to quantify the interference of salts and metal oxides, mixtures of SRM with known fractions of CC would help evaluate the relative impact of this source of measurement artifact.

\subsection{Manufacturer and user responsibilities}

Recommendation for manufacturer involvement: the companies that sell the instruments for measuring soot properties should partner with their clients to develop SRMs and improve the performance of their measurement systems. 
Instrument manufacturers have a major responsibility to serve their customers and the broader scientific community to help improve the quality of the measurements. These companies should be active participants in the process of developing SRMs appropriate for their measurement systems, then provide access to these reference materials so that instrument performance can be measured in a consistent way. In addition, they could provide an invaluable service by maintaining a database of results from instrument intercomparison studies. This would provide a historical range of comparison values among instruments and instrument types as well as information on the magnitude of the discrepancies related to the choice of reference materials and comparison method.

Recommendation for user involvement: users of soot measurement systems are responsible for (1) implementing the calibration and correction procedures accepted by the general community of those who use the instruments, (2) understanding their instruments and the reasons for performance variability, (3) keeping records of past instrument comparisons and performance checks and (4) participating in activities that improve the quality of the measurements.

The reference material workshop was a very positive step towards bringing together interested and concerned scientists whose desire was to move forward the efforts to find or develop SRMs that will improve the quality of data that we obtain from the present suite of instruments. The community of SP2 users (http://www.dropletmeasurement.com/ user-community.html) has set an example of the progress that can be made when users focus on a problem and reach agreement on how to solve it. There is a similar group of those who use filter-based instruments (see list of co-authors of Müller et al., 2011) to measure light absorption and who are presently interacting in an open forum to try and reach an agreement on how to correct for artifacts. This group needs to expand their efforts to find SRMs appropriate for their needs.

A large fraction of the workshop attendees are those who use TOA in their research and have published many articles describing the uncertainties and limitations that remain to be resolved. There is a strong need for a working group to be formed, similar to the group within CEN (Comite Européen de Normalisation), that will bring these researchers together to select or develop the SRMs that will help resolve these problems.

\section{Conclusions}

The large attendance at the soot workshop underscored the interest in the scientific community in the improvement of measurements of soot properties and highlighted the serious issues that are still impeding progress towards a better understanding of the formation and evolution of this type of particle that has such a large impact on climate and health.

The calibration, validation and intercomparison of techniques that measure soot properties require standard refer- ence materials (SRM) whose properties are related to the characteristic being measured. At this time there are no SRMs that meet all criteria established by internationally recognized organizations that set reference standards (with the exception of the German Standardization Organization for VDI/DIN that has accepted the spark-discharge carbon particle aerosol generator as a standard). The participants of the soot reference material workshop unanimously agreed that SRMs are needed for every measurement approach for evaluating soot properties. Although certified reference materials (CRM) are needed for some applications, only SRMs are seen as feasible at present.

A number of potential candidates for SRMs have been proposed for the three categories of instrumentation: light absorption, laser-induced incandescence and TOA. The user's group for SP2s is converging on accepted SRMs for their applications. Development of SRMs for light absorption and TOA will require a similar level of dedication and collaboration by users of the instruments that make these measurements.

A number of recommendations have evolved from the workshop that can help propel the selection or development of SRM. These recommendations do not require an inordinate amount of economic or human resources and can serve as legitimate motivation and justification for approaching funding agencies with requests to support these efforts to improve soot measurements.

Acknowledgements. The workshop on soot reference materials was sponsored by the Gesellschaft für Aerosolforschung (GAeF), Droplet Measurement Technologies, Sunset Laboratory and Magee Scientific. We would like to thank these organizations and businesses for their financial and moral support. We would also like to thank all of the participants for their valuable feedback during the meeting and afterwards during the preparation of this review manuscript. O. Popovicheva and M. Viana thank the Russian-Spain CSIC-RFBR 11-08-93989 project for SRM testing and travel support.

Edited by: W. Maenhaut

\section{References}

Arnott, W. P. and Moosmüller, H.: Method and apparatus for photoacoustic measurements, edited, Desert Research Institute, Reno, NV, USA, 2005.

Arnott, W. P., Moosmüller, H., Rogers, C. F., Jin, T., and Bruch, R.: Photoacoustic spectrometer for measuring light absorption by aerosols: Instrument description, Atmos. Environ., 33, 28452852, 1999.

Arnott, W. P., Moosmüller, H., and Walker, J. W.: Nitrogen dioxide and kerosene-flame soot calibration of photoacoustic instruments for measurement of light absorption by aerosols, Rev. Sci. Instrum., 71, 4545-4552, 2000. 
Arnott, W. P., Moosmüller, H., and Walker, J. W.: Photoacoustic instrument for measuring particles in a gas, edited, Patent 6,662,627, Desert Research Institute, Reno, Nev., 2003a.

Arnott, W. P., Moosmüller, H., Sheridan, P. J., Ogren, J. A., Raspet, R., Slaton, W. V., Hand, J. L., Kreidenweis, S. M., and Collett, J. L.: Photoacoustic and filter-based ambient aerosol light absorption measurements: Instrument comparisons and the role of relative humidity, J. Geophys. Res., 108, 4034, doi:10.1029/2002JD002165, 2003b.

Arnott, W. P., Hamasha, K., Moosmüller, H., Sheridan, P. J., and Ogren, J. A.: Towards Aerosol Light-Absorption Measurements with a 7-Wavelength Aethalometer: Evaluation with a Photoacoustic Instrument and 3-Wavelength Nephelometer, Aerosol Sci. Technol., 39, 17-29, 2005.

Bauschlicher Jr., C. W., Peeters, E., and Allamandola, L. J.: The infra-red spectra of very large, compact, highly symmetric, polycyclic aromatic hydrocarbons (PAHs), The Astrophysical Journal, 678, 316-327, 2008.

Birch, M. E. and Cary, R. A.: Elemental Carbon-Based Method for Monitoring Occupational Exposures to Particulate Diesel Exhaust, Aerosol Sci. Technol., 25, 221-241, 1996.

Bladt, H., Kireeva, E. Popovicheva, O., Timofeev, M., Heister, K., Uihlein, J., Ivleva, N., and Niessner, R.: Impact of Fe content in laboratory-produced soot aerosol on its composition, structure and thermo-chemical properties, submitted to Aerosol Sci. Technol, doi:10.1080/02786826.2012.711917, 2012.

Bond, T. C. and Bergstrom, R. W.: Light Absorption by Carbonaceous Particles: An Investigative Review, Aerosol Sci. Technol., 40, 27-67, 2006.

Bond, T. C., Anderson, T. L., and Campbell, D.: Calibration and Intercomparisonof Filter-Based Measurements of Visible Light Absorption by Aerosols, Aerosol Sci. Technol., 30, 582-600, 1999.

Boparai, P., Lee, J., and Bond, T. C.: Revisiting Thermal-Optical Analyses of Carbonaceous Aerosol Using a Physical Model. Aerosol Sci. Technol., 42, 930-948, 2008.

Braun, A.: Carbon speciation in airborne particulate matter with C(1s) NEXAFS spectroscopy, J. Environ. Monit., 7, 1059-1065, 2005.

Braun, A., Huggins, F. E., Kubatova, A., Wirick, S., Maricq, M. M., Mun, S. B., McDonald, J. D., Kelly, K. E., Shah, N., and Huffman, G. P.: Toward Distinguishing Woodsmoke and Diesel Exhaust in Ambient Particulate Matter, Environ. Sci. Technol., 42, 374-380, 2008.

Cali, J. P. and Reed, W. P.: Proceedings of the Symposium on Accuracy in Trace Analysis, NBS Spec. Publ. No. 422, Washington: US Government Printing Office, 41-63, 1976.

Cappa, C. D., Lack, D. A., Burkholder, J. B., and Ravishankara, A. R.: Bias in filter-based aerosol light absorption measurements due to organic aerosol loading: Evidence from laboratory measurements, Aerosol Sci. Technol., 42, 1022-1032, doi:10.1080/02786820802389285, 2008.

Casabianca, L. B., Shaibat, M. A., Cai, W. W., Park, S., Piner, R., and Ruoff, R. S.: NMR-Based Structural Modeling of Graphite Oxide Using Multidimensional ${ }^{13} \mathrm{C}$ Solid-State NMR and ab Initio Chemical Shift Calculations, J. Am. Chem. Soc., 124, 1393813948, 2010.

Castrucci, P., Tombolini, F., Scarselli, M., Scilletta, C., De Crescenzi, M., Diociaiuti, M., Casciardi, S., Rosei, F., and El
Khakani, M. A.: Comparison of the Local Order in Highly Oriented Pyrolitic Graphite and Bundles of Single-Wall Carbon Nanotubes by Nanoscale Extended Energy Loss Spectra, J. Phys. Chem. C, 113, 4848-4855, 2009.

Cavalli, F., Viana, M., Yttri, K. E., Genberg, J., and Putaud, J.-P.: Toward a standardised thermal-optical protocol for measuring atmospheric organic and elemental carbon: the EUSAAR protocol, Atmos. Meas. Tech., 3, 79-89, doi:10.5194/amt-3-79-2010, 2010.

CEN/TR 16243: Ambient air quality - Guide for the measurement of elemental carbon (EC) and organic carbon (OC) deposited on filters, Published by the European Committee for Standardization, ISBN- 9780580 740121, 34 pp., 2011.

Chen, L.-W. A., Chow, J. C., Watson, J. G., Moosmüller, H., and Arnott, W. P.: Modeling Reflectance and Transmittance of Quartz-Fiber Filter Samples Containing Elemental Carbon Particles: Implications for Thermal/Optical Analysis, J. Aerosol Sci., 35, 765-780, 2004.

Chow, J. C., Watson, J. G., Pritchett, L. C., Pierson, W. R., Frazier C. A., and Purcell, R. G.: The DRI Thermal Optical Reflectance Carbon Analysis System - Description, Evaluation and Applications in United-States Air-Quality Studies, Atmos. Environ. Part A - General Topics, 27, 1185-1201, 1993.

Chow, J. C., Watson, J. G., Crow, D., Lowenthal, D. H., and Merrifield, T.: Comparison of IMPROVE and NIOSH Carbon Measurements, Aerosol Sci. Technol., 34, 23-34, 2001.

Chow, J. C., Watson, J. G., Chen, L. W. A., Arnott, W. P., and Moosmuller, H.: Equivalence of Elemental Carbon by Thermal/Optical Reflectance and Transmittance with Different Temperature Protocols, Environ. Sci. Technol., 38, 4414-4422, 2004.

Conny, J. M., Klinedinst, D. B., Wight, S. A., and Paulsen, J. L.:Optimizing Thermal-Optical Methods for Measuring Atmospheric Elemental (black) Carbon: A Response Surface Study, Aerosol Sci. Technol., 37, 703-723, 2003.

CRC: 92nd Edition of the Handbook of chemistry and physics online, available at: http://www.hbcpnetbase.com/ (last access: 28 July 2012), 2011-2012.

Directive 2008/50/EC of the European Parliament and of the Council on ambient air quality and cleaner air for Europe, 21 May 2008, Official Journal of the European Union L, 152, 1-44, 2008.

Epstein, M. S.: The independent method concept for certifying chemical-composition reference materials, Spectrochimica Acta, 46, 1583-1591, 1991.

Fuller, K. A.: Scattering and Absorption Cross Sections of Compounded Spheres. I. Theory for External Aggregation, J. Opt. Soc. Am., 11, 3251-3260, 1994.

Fuller, K. A.: Scattering and Absorption Cross Sections of Compounded Spheres. II. Calculations For External Aggregation, J. Opt. Soc. Am. , 11, 881-892, 1995.

Fuller, K. A., Malm, W. C., and Kreidenweis, S. M.: Effects of Mixing on Extinction By Carbonaceous Particles, J. Geophys. Res., 104, 15941-15954, 1999.

Gao, R. S., Schwarz, J. P., Kelly, K. K., Fahey, D. W., Watts, L. A., Thompson, T. L., Spackman, J. R., Slowik, J. G., Cross, E. S., Han, J. H., Davidovits, P., Onasch, T. B., and Worsnop, D. R.: A novel method for estimating light-scattering properties of soot aerosols using a modified single-particle soot photometer, Aerosol Sci. Technol., 41, 125-135, 2007. 
Gysel, M., Laborde, M., Olfert, J. S., Subramanian, R., and Gröhn, A. J.: Effective density of Aquadag and fullerene soot black carbon reference materials used for SP2 calibration, Atmos. Meas. Tech., 4, 2851-2858, doi:10.5194/amt-4-2851-2011, 2011.

Hillebrand, W. F.: J. Ind. Eng. Chem., 8, 466, 1916.

Helsper, C., Moelter, W., Loeffler, F., Wadenpohl, C., Kaufmann, S., and Wenninger, G.: Investigations of a new aerosol generator for the production of carbon aggregate particles, Atmos. Environ., Part A: General Topics, 27A, 1271-1275, 1993.

Hitzenberger, R. M and Rosati, B.: Laboratory investigation of interferences in thermal and optical carbon analysis, International Aerosol Conference IAC 2010, Helsinki, Finland, $30 \mathrm{Au}-$ gust 2010, 2010.

IPCC (Intergovernmental Panel on Climate Change): Climate change 1995: The science of climate change, University Press, Cambridge, 1996.

ISO Guide 35:2006: Reference materials - General and statistical principles for certification, Published by the International Organization for Standardization, 2006

ISO Guide 30:1992/Amd 1:2008: Revision of definitions for reference material and certified reference material, Published by the International Organization for Standardization, 2008.

ISO Guide 34:2009: General requirements for the competence of reference material producers, Published by the International Organization for Standardization 2009.

Jankowski, N., Schmidl, C., Marr, I. L., Bauer, H., and Puxbaum, H.: Comparison of methods for the quantification of carbonate carbon in atmospheric $\mathrm{PM}_{10}$ aerosol samples, Atmos. Environ., 42, 8055-8064, 2008.

Johnson, R. L., Shah, J. J., Cary, R. A., and Huntzicker, J. J.: An Automated Thermal-Optical Method for the Analysis of Carbonaceous Aerosol. in "Atmospheric Aerosols", ACS Symposium Series, 16, Chapter 12, 223-233, 1981.

Karanasiou, A., Diapouli, E., Cavalli, F., Eleftheriadis, K., Viana, M., Alastuey, A., Querol, X., and Reche, C.: On the quantification of atmospheric carbonate carbon by thermal/optical analysis protocols, Atmos. Meas. Tech., 4, 2409-2419, doi:10.5194/amt4-2409-2011, 2011.

Kirchstetter, T. W. and Novakov, T.: Controlled generation of black carbon particles from a diffusion flame and applications in evaluating black carbon measurement methods, Atmos. Environ., 41, 1874-1888, 2007.

Klouda, G. A., Filliben, J. J., Parish, H. J., Chow, J. C., Watson, J. G., and Cary, R. A.: Reference Material 8785: Air Particulate Matter on Filter Media, Aerosol Sci. Technol., 39, 173-183, 2005.

Kondo, Y., Sahu, L., Kuwata, M., Miyazaki, Y., Takegawa, N., Moteki, N., Imaru, J., Han, S., Nakayama, T., Oanh, K. N. T., Hu, M., Kim, Y. J., and Kita, K: Stabilization of the filter-based absorption photometry by the use of a heated inlet, Aerosol Sci. Technol., 43, 741-756, 2009.

Laborde, M., Mertes, P., Zieger, P., Dommen, J., Baltensperger, U., and Gysel, M.: Sensitivity of the Single Particle Soot Photometer to different black carbon types, Atmos. Meas. Tech., 5, 10311043, doi:10.5194/amt-5-1031-2012, 2012a.

Laborde, M., Schnaiter, M., Linke, C., Saathoff, H., Naumann, K.H., Möhler, O., Berlenz, S., Wagner, U., Taylor, J. W., Liu, D., Flynn, M., Allan, J. D., Coe, H., Heimerl, K., Dahlkötter, F., Weinzierl, B., Wollny, A. G., Zanatta, M., Cozic, J., Laj, P.,
Hitzenberger, R., Schwarz, J. P., and Gysel, M.: Single Particle Soot Photometer intercomparison at the AIDA chamber, Atmos. Meas. Tech. Discuss., 5, 3519-3573, doi:10.5194/amtd-5-35192012, 2012 b.

Lack, D. A., Lovejoy, E., Baynard, T., Pettersson, A., and Ravishankara, A. R.: Aerosol Absorption Measurement using Photoacoustic Spectroscopy: Sensitivity, Calibration, and Uncertainty Developments, Aerosol Sci. Technol., 40, 697-708, 2006.

Lack, D. A., Cappa, C. D., Covert, D.S., Baynard, T., Massoli, P., Sierau, B., Bates, T. S., Quinn, P. K., Lovejoy, E. R., and Ravishankara, A. R.: Bias in filter-based aerosol light absorption measurements due to organic aerosol loading: Evidence from ambient measurements, Aerosol Sci. Technol., 42, 1033-1041, 2008.

Lack, D. A., Cappa, C. D., Cross, E. S., Massoli, P., Ahern, A. T., Davidovits, P., and Onasch, T. B.: Absorption Enhancement of Coated Absorbing Aerosols: Validation of the Photo-Acoustic Technique for Measuring the Enhancement, Aerosol Sci. Technol., 43, 1006-1012, 2009.

Lewis, K., Arnott, W. P., Moosmüller, H., and Wold, C. E.: Strong spectral variation of biomass smoke light absorption and single scattering albedo observed with a novel dual-wavelength photoacoustic instrument, J. Geophys. Res., 113, D16203, doi:10.1029/2007JD009699, 2008

Moteki, N. and Kondo, Y.: Effects of Mixing State on Black Carbon Measurements by Laser-Induced Incandescence, Aerosol Sci. Technol., 41, 398-417, 2007.

Moteki, N. and Kondo, Y.: Dependence of Laser-Induced Incandescence on Physical Properties of Black Carbon Aerosols: Measurements and Theoretical Interpretation, Aerosol Sci. Technol., 44, 663-675, 2010.

Moteki, N., Kondo, Y., Nakayama, T., Kita, K., Sahu, L. K., Ishigai, T., Kinase, T., and Matsumi, Y.: Radiative transfer modeling of filter-based measurements of light absorption by particles: Importance of particle size dependent penetration depth, J. Aerosol Sci., 41, 401-412, 2010.

Müller, T., Henzing, J. S., de Leeuw, G., Wiedensohler, A., Alastuey, A., Angelov, H., Bizjak, M., Collaud Coen, M., Engström, J. E., Gruening, C., Hillamo, R., Hoffer, A., Imre, K., Ivanow, P., Jennings, G., Sun, J. Y., Kalivitis, N., Karlsson, H., Komppula, M., Laj, P., Li, S.-M., Lunder, C., Marinoni, A., Martins dos Santos, S., Moerman, M., Nowak, A., Ogren, J. A., Petzold, A., Pichon, J. M., Rodriquez, S., Sharma, S., Sheridan, P. J., Teinilä, K., Tuch, T., Viana, M., Virkkula, A., Weingartner, E., Wilhelm, R., and Wang, Y. Q.: Characterization and intercomparison of aerosol absorption photometers: result of two intercomparison workshops, Atmos. Meas. Tech., 4, 245-268, doi:10.5194/amt-4-245-2011, 2011

Nakayama, T., Kondo, Y., Moteki, N., Sahu, L. K., Kinase, T., Kita, K., and Matsumi, Y.: Size-dependent correction factors for absorption measurements using filter-based photometers: PSAP and COSMOS, J. Aerosol Sci., 41, 333-343, 2010.

National Institute of Occupational Safety and Health (NIOSH): ElementalC arbon (Diesel Particulate): Method 5040, Issue 3 (Interim), NIOSH Manual of Analytical Methods. , 4th Edn, DHHS (NIOSH) Publication No. 94-113, edited by: Cassinelli, M. E. and O'Connor, P. F., National Institute for Occupational Safety and Health, US Department of Health and Human Services, Cincinnati, OH, 1999. 
Niessner, R. and Helsper, C.: Application of a multistep condensation nuclei counter as a detector for particle surface composition, J. Aerosol Sci., 16, 201-209, 1985.

Novakov, T. and Corrigan, C. E.: Thermal Characterization of Biomass Smoke Particles, Mikrochim Acta, 119, 157-166, 1995.

Ogren, J. A.: Comment on "Calibration and intercomparison of filter-based measurements of visible light absorption by aerosols", Aerosol Sci. Technol., 44, 589-591, 2010.

Petzold, A. and Niessner, R.: In Situ Measurements on Carbon Aerosols with Photoacoustic Spectroscopy, SPIE, 1716, 510516, 1992.

Petzold, A. and Schönlinner, M.: Multi-angle absorption photometry - a new method for the measurement of aerosol light absorption and atmospheric black carbon, J. Aerosol Sci., 35, 421-441, 2004.

Petzold, A., Schloesser, H., Sheridan, P. J., Arnott, W. P., Ogren, J. A., and Virkkula, A.: Evaluation of multiangle absorption photometry for measuring aerosol light absorption, Aerosol Sci. Technol., 39, 40-51, 2005.

Piazzalunga, A., Bernardoni, V., Fermo, P., Valli, G., and Vecchi, R.: Technical Note: On the effect of water-soluble compounds removal on EC quantification by TOT analysis in urban aerosol samples, Atmos. Chem. Phys., 11, 10193-10203, doi:10.5194/acp-11-10193-2011, 2011.

Popovicheva, O. B., Persiantseva, N. M., Kuznetsov, B. V., Rakhmanova, T. A. ,Shonija, N. K., Suzanne, J., and Ferry, D.: Microstructure and water adsorbability of aircraft combustor and kerosene flame soots: Toward an aircraft generated soot laboratory surrogate, J. Phys. Chem., 107, 10046-10054, 2003.

Popovicheva, O. B., Baumgardner, D., Subramanian, R., Kok, G., Cary, R., Vlasenko, E., Khokhlova, T., Shonija, N., and Kireeva, E.: Tailored graphitized soot as reference material for EC/OC measurement validation, Atmos. Meas. Tech., 4, 923932, doi:10.5194/amt-4-923-2011, 2011.

Robles, J., Chow, J. C., and Watson, J. G.: Carbonate and mineral interference during thermal optical analysis, Proceedings of the 10th International Conference on Carbonaceous Particles in the Atmosphere, 26-29 June 2011, Vienna, Austria, 2011.

Roth, C. Ferron, G. A., Karg, E., Lentner, B., Schumann, G., Takenaka, S., and Heyder, J.: Generation of Ultrafine Particles by Spark Discharging, Aerosol Sci. Technol., 38, 228-235, 2004.

Schauer, J. J., Mader, B. T., Deminter, J. T., Heidemann, G., Bae, M. S., Seinfeld, J. H., Flagan, R. C., Cary, R. A., Smith, D., Huebert, B. J., Bertram, T., Howell, S., Kline, J. T., Quinn, P., Bates, T., Turpin, B., Lim, H. J., Yu, J. Z., Yang, H., and Keywood, M. D.: ACE-Asia Intercomparison of a Thermal-Optical Method for the Determination of Particle-Phase Organic and Elemental Carbon, Environ. Sci. Technol., 37, 993-1001, 2003.

Schmid, H., Laskus, L., Abraham, H. J., Baltensperger, U., Lavanchy, V., Bizjak, M., Burba, P., Cachier, H., Crow, D., Chow, J., Gnauk, T., Even, A., ten Brink, H. M., Giesen, K. P., Hitzenberger, R., Hueglin, E., Maenhaut, W., Pio, C., Carvalho, A., Putaud, J. P., Toom-Sauntry, D., and Puxbaum, H.: Results of the "Carbon Conference" International Aerosol Carbon Round Robin Test Stage I, Atmos. Environ., 35, 2111-2121, 2001.

Schmid, J., Grob, B., Niessner, R., and Ivleva, N: Multi-Wavelength Raman Microspectroscopy for Rapid Prediction of Soot Oxidation Reactivity, Anal. Chem., 83, 1173-1179, 2011.
Schmidt-Rohr, K. and Mao, J.-D.: Efficient CH-Group Selection and Identification in 13C Solid-State NMR by Dipolar DEPT and 1H Chemical-Shift Filtering, J. Am. Chem. Soc., 124, 1393813948, 2002.

Schmidt, M. W. I., Skjemstad, J. O., Czimczik, C. I., Glaser, B., Prentice, K. M., Gelinas, Y., and Kuhlbusch, T. A. J.: Comparative analysis of black carbon in soils, Global Biogeochem. Cy., 15, 163-167, 2001.

Schmidt, M. W. I., Masiello, C. A., and Skjemstad, J. O.: Final recommendations for reference materials in black carbon analysis, EOS, 84, 582-583, 2003.

Schwarz, J. P., Gao, R. S., Fahey, D. W., Thomson, D. S., Watts, L. A.,Wilson, J. C., Reeves, J. M., Darbeheshti, M., Baumgardner, D. G., Kok, G. L., Chung, S. H., Schulz, M., Hendricks, J., Lauer, A., Kärcher, B., Slowik, J. G., Rosenlof, K. H., Thompson, T. L., Langford, A. O., Loewenstein, M., and Aikin, K. C.: Single-particle measurements of midlatitude black carbon and light-scattering aerosols from the boundary layer to the lower stratosphere, J. Geophys. Res., 111, D16207, doi:10.1029/2006JD007076, 2006.

Seinfeld, J. H. and Pandis, S. N.: Atmospheric Chemistry and Physics, from Air Pollution to Climate Change, John Wiley and Sons, New York., 1326 pp., 1998.

Slowik, J. G., Cross, E. S., Han, J.-H., Davidovits, P., Onasch, T. B., Jayne, J. T., Williams, L. R., Canagaratna, M. R., Worsnop, D. R., Chakrabarty, R. K., Moosmuller, H., Arnott, W. P., Schwarz, J. P., Gao, R.-S., Fahey, D. W., Kok, G. L., and Petzold, A.: An InterComparison of Instruments Measuring Black Carbon Content of Soot Particles, Aerosol Sci. Technol., 41, 295-314, 2007.

Spanner, G., Schroeder, H., Petzold, A., and Niessner, R.: Generation of carbon aerosols by fragmentation of acetylene in a laserinduced plasma, J. Aerosol Sci., 25, 265-275, 1994.

Stephens, M., Turner, N., and Sandberg, J.: Particle identification by laser-induced incandescence in a solid-state laser cavity, Appl. Opt., 42, 3726-3736, 2003.

Stipte, C. B., Higgins, B. S., Lucas, D., Koshland, C. P., and Sawyer, R. F.: Inverted co-flow diffusion flame for producing soot, Rev. Sci. Instr., 76, 023908, doi:10.1063/1.1851492, 2005.

Subramanian, R., Khlystov A. Y., and Robinson, A. L.: Effect of Peak Inert-Mode Temperature on Elemental Carbon Measured using Thermal-optical Analysis, Aerosol Sci. Technol., 40, 763780, 2006.

Subramanian, R., Roden, C. A., Boparai, P., and Bond, T. C.: Yellow beads and missing particles: Trouble ahead for filter based absorption measurements, Aerosol Sci. Technol., 41, 630-637, doi:10.1080/02786820701344589, 2007.

Thompson, J. E., Barta, N., Policarpio, D., and DuVall. R.: A fixed frequency aerosol albedometer, Opt. Express, 16, 2191-2205, 2008.

Turpin, B. J., Cary, R. A., and Huntzicker, J. J.: An In Situ, TimeResolved Analyzer for Aerosol Organic and Elemental Carbon, Aerosol Sci. Technol., 12, 161-171, 1990.

Virkkula, A.: Correction of the Calibration of the 3-wavelength Particle Soot Absorption Photometer (3 PSAP), Aerosol Sci. Technol., 44, 706-712, 2010.

Virkkula, A., Ahlquist, N. C., Covert, D. S., Arnott, W. P., Sheridan, P. J., Quinn, P. K., and Coffman, D. J.: Modification, calibration and a field test of an instrument for measuring light absorption by particles, Aerosol Sci. Technol., 39, 68-83, 2005. 
Virkkula, A., Mäkelä, T., Yli-Tuomi, T., Hirsikko, A., Koponen, I.K., Häameri, K., and Hillamo, R.: A simple procedure for correcting loading effects of aethalometer data, J. Air Waste Manage., 57, 1214-1222, doi:10.3155/1047-3289.57.10.1214, 2007.

Weingartner, E., Saathoff, H., Schnaiter, M., Streit, N., Bitnar, B., and Baltensperger, U.: Absorption of light by soot particles: determination of the absorption coefficient by means of aethalometers, J. Aerosol Sci., 34, 1445-1463, 2003.

Wang, Y., Chung, A., and Paulson, S. E.: The effect of metal salts on quantification of elemental and organic carbon in diesel exhaust particles using thermal-optical evolved gas analysis, Atmos. Chem. Phys., 10, 11447-11457, doi:10.5194/acp-1011447-2010, 2010.
Yang, H. S. and Yu, J.: Uncertainties in Charring Correction in the Analysis of Elemental and Organic Carbon in Atmospheric Particles by Thermal/Optical Methods, Environ. Sci. Technol., 36, 5199-5124, 2002.

Yolken, H. T.: Proceedings of the 6th Materials Research Symposium, in: NBS Spec. Publ. 408, edited by: Seward, R.D., NBS, Gaithersburg, MD, 1973.

Yu, J. Z., Xu, J. H., and Yang, H.: Charring Characteristics of Atmospheric Organic Particulate Matter in Thermal Analysis, Environ. Sci.Technol., 36, 754-761, 2002. 